\title{
Zero and negative eigenvalues of the conformal Laplacian
}

Article

Accepted Version

Gower, A. R., Hassannezhad, A., Jakobson, D. and Levitin, M. (2016) Zero and negative eigenvalues of the conformal Laplacian. Journal of Spectral Theory, 6 (4). pp. 793-806. ISSN 1664-0403 doi: https://doi.org/10.4171/JST/142 Available at https://centaur.reading.ac.uk/65980/

It is advisable to refer to the publisher's version if you intend to cite from the work. See Guidance on citing.

To link to this article DOI: http://dx.doi.org/10.4171/JST/142

Publisher: European Mathematical Society

All outputs in CentAUR are protected by Intellectual Property Rights law, including copyright law. Copyright and IPR is retained by the creators or other copyright holders. Terms and conditions for use of this material are defined in the End User Agreement.

\section{www.reading.ac.uk/centaur}

\section{CentAUR}

Central Archive at the University of Reading

Reading's research outputs online 


\title{
ZERO AND NEGATIVE EIGENVALUES OF THE CONFORMAL LAPLACIAN
}

\author{
A.R. GOVER, A. HASSANNEZHAD, D. JAKOBSON, AND M. LEVITIN \\ This paper is dedicated to the memory of Yuri Safarov
}

\begin{abstract}
We show that zero is not an eigenvalue of the conformal Laplacian for generic Riemannian metrics. We also discuss non-compactness for sequences of metrics with growing number of negative eigenvalues of the conformal Laplacian.
\end{abstract}

\section{INTRODUCTION}

In [CGJP14, CGJP13], the authors studied spectra and eigenfunctions of conformally covariant operators on compact manifolds of dimension $n \geq 3$. They showed, in particular, that the number of negative eigenvalues of conformal Laplacian is unbounded on any such manifold. One of the questions left open in those papers was whether for a generic Riemannian metric $g$ on a compact $n$-dimensional manifold $M, 0$ is an eigenvalue of the conformal Laplacian $Y_{g}:=-\Delta_{g}+c_{n} R_{g}$. The operator $Y_{g}$ is also called the Yamabe operator. Here $-\Delta_{g}$ is the nonnegative-definite Laplacian for the metric $g, c_{n}:=(n-2) /(4(n-1))$, and $R_{g}$ denotes the scalar curvature of $g$. In this paper we address that question.

Our first main result is

Theorem 1.1. For generic smooth metrics $g$ on $M$, zero is not an eigenvalue of $Y_{g}$.

It follows from a transformation formula for $Y_{g}$ that if 0 is an eigenvalue of $Y_{g_{0}}$, then it is also an eigenvalue of $Y_{g_{1}}$ for all metrics $g_{1}$ in the conformal class [ $\left.g_{0}\right]$. Accordingly, one needs to change conformal class to find metrics for which 0 is not an eigenvalue of $Y_{g}$.

Also, 0 is an eigenvalue of $Y_{g}$ for conformally scalar flat metrics $g$, i.e. those metrics lying in a conformal class $\left[g_{0}\right]$ of a scalar-flat metric $g_{0}$, such that $R_{g_{0}} \equiv 0$. The corresponding eigenfunction $u$ is given by $u\left(g_{0}\right) \equiv 1$, and by a suitable power of the conformal factor one obtains the eigenfunction for $g \in\left[g_{0}\right]$.

It is also clear that 0 is not an eigenvalue of $Y_{g}$ for metrics $g$ with positive scalar curvature $R_{g}$, and hence in the corresponding conformal classes. However, it is known that some manifolds do not admit metrics with positive scalar curvature, [KW75]. Accordingly, in the current paper we restrict ourselves to metrics lying in conformal classes of metrics with negative scalar curvature.

2010 Mathematics Subject Classification. 58J50, 58J37, 58D17, 53A30.

Key words and phrases. Spectral geometry, conformal geometry, conformal Laplacian, eigenvalue 0 , negative eigenvalues, generic metrics, manifolds of metrics, pre-compactness.

A.R.G. gratefully acknowledges support from the Royal Society of New Zealand via Marsden Grant 13-UOA-018; D.J. was supported by NSERC, FQRNT and Peter Redpath fellowship. 
Remark 1.2. We remark that our proof of Theorem 1.1 works verbatim to show an analogous statement for operators $P_{g, c}=\Delta_{g}+c R_{g}$, where $c \in \mathbf{R}$ is any constant satisfying $c \neq 0, c \neq 1 / 2$.

The crucial result is Proposition 4.1, and the only part of the proof where the numerical value of $c$ becomes important is the argument after the equation (3), where it is necessary that $2 c-1 \neq 0$, hence $c \neq 1 / 2$. Also, we assume that the corresponding eigenfunctions are orthogonal to constants, hence we require that $c \neq 0$. Note that, $P_{g, c}$ is only conformally covariant for $c=c_{n}$, so for other values of $c$ an argument using conformal perturbations (as in [BW80]) should also work. The case $c=0$ corresponds to the very-well studied of eigenvalues of the Laplacian $\Delta_{g}$.

Perturbation theory of conformally covariant operators was previously considered in [Can14, Pon]; see also [Mai97, Dah03, Dah08] for the corresponding results for the Dirac operator. Applications to eigenvalue multiplicity of nonzero eigenvalues were discussed in [Can14]; arbitrary eigenvalues were considered in [Pon], but the question of whether $\operatorname{ker} Y_{g}$ is generically empty was not settled. It seems interesting to understand whether 0 is generically a simple eigenvalue of $Y_{g}$, among those metrics for which it is an eigenvalue of $Y_{g}$.

Another question considered in this paper concerns the study of sequence of metrics $g_{k}$ such that the number of negative eigenvalues of $Y_{g_{k}}$ increases. Recall that it was shown in [CGJP14] that the results in [Loh96] imply that the number of negative eigenvalues of $Y_{g}$ can become arbitrarily large for metrics $g$ on any compact manifold of dimension $\geq 3$; thus, it seems natural to ask what is the geometric significance of the increasing number of negative eigenvalues of $Y_{g}$.

In Section 5, we show that a sequence of metrics $g_{k}$, such that the number of negative eigenvalues of $Y_{g_{k}}$ increases, cannot satisfy two natural "pre-compactness" conditions (see Proposition 5.3).

\section{The SPACE of CONFORMAL STRUCTURES}

Let $M$ be a compact orientable manifold of dimension $n \geq 3$; we denote by $\mathcal{M}$ the space of all Riemannian metrics on $M$. For simplicity, we only consider $C^{\infty}$ metrics on $M$, although the regularity can be lowered significantly.

Definition 2.1. Given $k \geq 1$, we denote by $\mathcal{M}_{0, k}$ the set of all metrics $g$ on $M$ s.t. the multiplicity of 0 as an eigenvalue of $Y_{g}$ is at least $k$.

As we remarked in section 1 , if $g_{0} \in \mathcal{M}_{0, k}$, then so is every metric $g$ in the conformal class $\left[g_{0}\right]$; also that condition is invariant under composition with diffeomorphisms of $M$. Consider the action on $\mathcal{M}$ of the group $\mathcal{P}$ of (pointwise) conformal transformations (multiplication by positive functions), as well as by the group $\mathcal{D}$ of diffeomorphisms; we shall denote by $\mathcal{D}_{0}$ the subgroup of $\mathcal{D}$ of diffeomorphisms isotopic to identity. It seems natural to consider the Teichmüller space of conformal structures

$$
\mathcal{T}(M)=\frac{\mathcal{M} / \mathcal{P}}{\mathcal{D}_{0}},
$$

or the Riemannian moduli space of conformal structures

$$
\mathcal{R}(M)=\frac{\mathcal{M} / \mathcal{P}}{\mathcal{D}}
$$


in the terminology of Fischer and Monkrief, [FM96, FM97]. ${ }^{1}$

Definition 2.2. We denote by $\mathcal{T}_{0, k}(M)$ the Teichmüller space of conformal structures corresponding to metrics $g_{0} \in \mathcal{M}_{0, k}$, i.e. the projection of $\mathcal{M}_{0, k}$ into $\mathcal{T}(M)$.

The meaning of Theorem 1.1 is the following, and we prove this in Section 4

Theorem 2.3. The complement $\mathcal{T}_{0,1}^{c}$ of the set $\mathcal{T}_{0,1}(M)$ in $\mathcal{T}(M)$ is open and dense in $\mathcal{T}(M)$.

\section{Curves of metrics}

Let $g_{0}$ be a metric on $M$ such that 0 is an eigenvalue of $Y_{g_{0}}$ with multiplicity $m$, so, $g_{0} \in \mathcal{M}_{0, m}$ (recall the definition 2.1). We note that it was shown in [BD03, Lemma 3.4] that the eigenvalues of $Y_{g}$ depend continuously on $g$ in the $C^{1}$-topology (see also [KS60]). Thus, $\mathcal{M}_{0, m_{2}}$ is a closed subset of $\mathcal{M}_{0, m_{1}}$ for $0 \leq m_{1}<m_{2}$, in the $C^{k}$ topology for any $k \geq 1$. Let $C^{\omega}\left(I_{\epsilon}, \mathcal{M}_{0, m}\right), \epsilon>0$ be the space of analytic curves of metrics $g(t), t \in I_{\epsilon}=(-\epsilon, \epsilon)$ in $\mathcal{M}_{0, m}$ for $m \geq 1$. We would like to study the space

$$
T_{g_{0}}\left(\mathcal{M}_{0, m}\right)=\left\{h \in S^{2}(M): \exists g(t) \in C^{\omega}\left(I_{\epsilon}, \mathcal{M}_{0, m}\right), \epsilon>0, \text { with } \dot{g}(0)=h\right\},
$$

where $S^{2}(M)$ is the space of symmetric 2-tensors on $M$.

Denote by $E_{0}$ the zero eigenspace of $Y_{g_{0}}$; it has dimension $m$. Let $\Pi_{0}$ denote the orthogonal projection into $E_{0}$ with respect to $L^{2}\left(M, d V_{g_{0}}\right)$. Consider a curve $g_{t}$ of metrics on $M$ passing through $g_{0}$ at $t=0$; denote the $t$-derivative by *

Let $\dot{g}(0)=h$, i.e. $g(t)=g_{0}+t h+o(t)$. We denote by $Q_{g_{0}, h}$ the operator

$$
Q_{g_{0}, h}:=\Pi_{0} \dot{Y}_{g}=\Pi_{0}\left(c_{n} \dot{R}-\dot{\Delta}\right): E_{0} \rightarrow E_{0},
$$

Sometimes when the dependence on the metric $g_{0}$ is clear, we shall omit the subscript $g_{0}$ and simply write $Q_{h}$.

We have the following:

Proposition 3.1. The space $T_{g_{0}}\left(\mathcal{M}_{0, k}\right)$ consists of all the tensors

$$
\mathcal{H}_{0, k}:=\left\{h: 0 \text { is an eigenvalue of } Q_{g_{0}, h} \text { of multiplicity } \geq k\right\} \text {. }
$$

Proof of Proposition 3.1. We refer to [Rel69, page 74] and the discussion in $[$ Can14, $\S 4,5]$ for basic results about the perturbation theory of conformally covariant operators; see also [DWW05], where some important formulas that we use in our argument were derived.

It follows from general theory that for a real-analytic family of self-adjoint operators $\left\{Y_{g(t)}\right\}$, eigenvalue and eigenfunction branches can be chosen to depend analytically on the perturbation parameter $t$, for $t$ small enough, see for example [Ber73, Lemma 3.15]. Moreover, there is a positive constant $\epsilon$ such that, for $t$ small enough, the number of eigenvalues of $Y_{g(t)}$ in the interval $(-\epsilon, \epsilon)$ is equal to $m$, where $m \geq k$ is the multiplicity of 0 for $Y_{g_{0}}$; and the eigenvalue derivatives are equal to the eigenvalues of $Q_{g_{0}, h}$. In particular, $Q_{g_{0}, h} \equiv 0$ for any real-analytic perturbation of $g_{0}$ when $m=k$. The result is now immediate from the definition of $T_{g_{0}}\left(\mathcal{M}_{0, k}\right)$ and $\mathcal{H}_{0, k}$.

\footnotetext{
${ }^{1}$ If $M$ is an orientable two-dimensional manifold, then $\mathcal{T}(M)$ (resp. $\left.\mathcal{R}(M)\right)$ are the usual Teichmüller (resp. moduli) spaces. In [FM97], the space $\mathcal{T}(M)$ for Haken 3-manifolds $M$ of degree 0 is proposed as a configuration space for a Hamiltonian reduction of Einstein's vacuum field equations.
} 
It is well-known (see e.g. [BE69, FM77]) that the tangent space to $\mathcal{T}(M)$ at $g_{0}$ may be identified with the space of all transverse traceless symmetric tensors $h$ satisfying $\operatorname{tr}_{g_{0}} h=0, \delta h=0$. Clearly, the projection of $\mathcal{H}_{0, k}$ into the tangent space of $\mathcal{T}(M)$ at $g_{0}$, consists of all transverse traceless tensors lying in $\mathcal{H}_{0, k}$.

\section{Nullspace of $Y_{g}$}

In this section we prove Theorem 2.3. We keep the notation from section 3. For convenience we shall assume that $g_{0}$ is a Yamabe metric, i.e. that $R_{g_{0}} \equiv-1$.

Theorem 2.3 will follow from the following important result:

Proposition 4.1. There exists a symmetric tensor $h$ such that $Q_{g_{0}, h} \not \equiv 0$.

We postpone the proof that Proposition 4.1 implies Theorem 2.3 until later, and first prove the Proposition.

\section{Proof of Proposition 4.1.}

Let $g_{t}$ be a curve of metrics real-analytic in $t$, and $\psi \not \equiv 0$ be an element of $E_{0}$ that belongs to the Rellich basis of $g_{t}$, i.e. $\psi$ is an eigenvector of the operator $Q_{h}$ defined in (1).

Differentiating the eigenfunction equation

$$
\left(-\Delta+c_{n} R\right) \psi=\lambda \psi,
$$

we find that

$$
\dot{\lambda} \psi=\left(-\Delta-\lambda+c_{n} R\right) \dot{\psi}+\left(c_{n} \dot{R}-\dot{\Delta}\right) \psi
$$

It suffices to show that there exists a metric deformation $g_{t}$ real-analytic in $t$ such that $\dot{\lambda} \neq 0$.

Take the inner product (with respect to $d V_{g_{0}}$ ) of both sides with $\psi$. Since $(-\Delta-$ $\left.\lambda+c_{n} R\right)$ is self-adjoint, we find that

$$
\left(\left(-\Delta-\lambda+c_{n} R\right) \dot{\psi}, \psi\right)=\left(\dot{\psi},\left(-\Delta-\lambda+c_{n} R\right) \psi\right)=0 .
$$

Then

$$
\dot{\lambda}(\psi, \psi)=\left(\left(c_{n} \dot{R}-\dot{\Delta}\right) \psi, \psi\right) .
$$

We assume for contradiction that $\dot{\lambda}=0$ for any analytic perturbation $g_{t}$, i.e. that (1) is identically zero for any analytic perturbation $g_{t}$.

We next give the expressions for $\dot{\Delta}$ and $\dot{R}$. We need to recall some notation. Let $C^{\infty}\left(\otimes^{r} T^{*} M\right)$ be the space of $(r, 0)$-tensors on $M$, and $C^{\infty}(M)=C^{\infty}\left(\otimes^{0} T^{*} M\right)$. We consider the covariant derivative

$$
\nabla: C^{\infty}\left(\otimes^{r} T^{*} M\right) \rightarrow C^{\infty}\left(\otimes^{r+1} T^{*} M\right)
$$

which in local coordinates is given by

$$
\nabla \alpha=\sum_{i} \nabla_{i} \alpha \otimes d x_{i}
$$

Notice that $d=\nabla: C^{\infty}(M) \rightarrow C^{\infty}\left(T^{*} M\right)$. We denote the formal adjoint of $\nabla$ by

$$
\delta: C^{\infty}\left(\otimes^{r+1} T^{*} M\right) \rightarrow C^{\infty}\left(\otimes^{r} T^{*} M\right),
$$

i.e. for every $\alpha \in C^{\infty}\left(\otimes^{r} T^{*} M\right)$ and $\beta \in C^{\infty}\left(\otimes^{r+1} T^{*} M\right),(\nabla \alpha, \beta)=(\alpha, \delta \beta)$. Here, $(\cdot, \cdot)=\int_{M}\langle\cdot, \cdot\rangle$, where $\langle\cdot, \cdot\rangle$ is the pointwise inner product. We can now 
recall the expressions for $\dot{\Delta}$ and $\dot{R}$ computed in [DWW05, (2.5) and (2.6)], see also [Ber70, Ber73]. They are,

$$
\dot{R}=-\langle h, \operatorname{Ric}\rangle+\delta^{2} h-\Delta \operatorname{tr} h,
$$

and

$$
\dot{\Delta} f=-\left\langle h, \nabla^{2} f\right\rangle+\left\langle\delta h+\frac{1}{2} d \operatorname{tr} h, d f\right\rangle,
$$

where $\nabla^{2} f$ is the Hessian of $f$, and $\delta^{2}$ is the formal adjoint of the Hessian. Recall the pointwise inner product on $C^{\infty}\left(\otimes^{2} T^{*} M\right)$ is

$$
\langle\alpha, \beta\rangle=\sum_{i, j} \alpha^{i j} \beta_{i j}
$$

in particular, $\operatorname{tr}_{g} h=\langle g, h\rangle$. We know apriori that the only metric deformations that will change the eigenvalue 0 , are transverse traceless deformations $h$ of $g$.

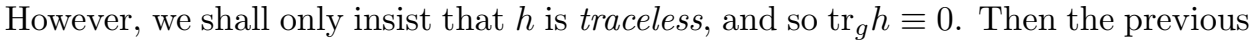
expressions simplify to

$$
\dot{R}=-\langle h, \operatorname{Ric}\rangle+\delta^{2} h
$$

and

$$
\dot{\Delta} f=-\left\langle h, \nabla^{2} f\right\rangle+\langle\delta h, d f\rangle .
$$

Let $A:=\dot{\lambda}(\psi, \psi)$. Combining the above expressions, we find that

$$
\begin{aligned}
A & =\left(\left\langle h, \nabla^{2} \psi-c_{n} \psi \mathrm{Ric}\right\rangle-\langle\delta h, d \psi\rangle+c_{n} \psi \delta^{2} h, \psi\right) \\
& =\left(h, \psi \nabla^{2} \psi\right)-c_{n}\left(h, \psi^{2} \mathrm{Ric}\right)-(h, \nabla(\psi d \psi))+c_{n}\left(\psi \delta^{2} h, \psi\right) \\
& =\left(h, \psi \nabla^{2} \psi\right)-c_{n}\left(h, \psi^{2} \mathrm{Ric}\right)-\left(h, \psi \nabla^{2} \psi\right)-(h, d \psi \otimes d \psi)+c_{n}\left(\psi \delta^{2} h, \psi\right) \\
& =-\left(h, c_{n} \psi^{2} \mathrm{Ric}\right)-(h, d \psi \otimes d \psi)+\left(\delta^{2} h, c_{n} \psi^{2}\right) \\
& =\int_{M}\left\langle h, c_{n}\left(\nabla^{2} \psi^{2}-\psi^{2} \mathrm{Ric}\right)-d \psi \otimes d \psi\right\rangle \\
& =\int_{M}\left\langle h, c_{n} \psi\left(2 \nabla^{2} \psi-\psi \mathrm{Ric}\right)+\left(2 c_{n}-1\right) d \psi \otimes d \psi\right\rangle .
\end{aligned}
$$

To get the last equality, we use the identity $\nabla^{2} \psi^{2}=2\left(\psi \nabla^{2} \psi+d \psi \otimes d \psi\right)$. Using the assumption that $\operatorname{tr}_{g} h=\langle g, h\rangle=0$, we find that

$$
A=\int_{M}\left\langle h, c_{n} \psi\left(2 \psi \stackrel{\circ}{\nabla}^{2} \psi-\psi^{2} \text { Ric }\right)+\left(2 c_{n}-1\right)(d \psi \otimes d \psi)^{o}\right\rangle,
$$

where $\stackrel{\circ}{V}=V-\frac{1}{n} \operatorname{tr}_{g} V g$ is the traceless part of the corresponding expression $V$.

Putting $h=c_{n} \psi\left(2 \psi \stackrel{\nabla}{ }^{2} \psi-\psi^{2}\right.$ Ric) $+\left(2 c_{n}-1\right)(d \psi \otimes d \psi)^{o}$ (which is symmetric and traceless), we find that $A=0$ if and only if

$$
c_{n} \psi\left(2 \psi \stackrel{\circ}{ }^{2} \psi-\psi^{2} \text { Ric }\right)+\left(2 c_{n}-1\right)(d \psi \otimes d \psi)^{o} \equiv 0 .
$$

We next remark that equation (3) has no non-trivial solutions. Indeed, take $g_{0}$ to be the Yamabe metric; recall we assume that $R_{g_{0}} \equiv-1$. By assumption $\psi_{g_{0}}$ is an eigenfunction of $\Delta_{g_{0}}$ with eigenvalue $c_{n}$, hence it is $L^{2}$-orthogonal to the constant function, and changes sign on $M$. Let $\mathcal{N}(\psi)$ denote the nodal set of $\psi$. The term $c_{n} \psi\left(2 \psi \dot{\nabla}^{2} \psi-\psi^{2}\right.$ Ric $)$ vanishes on $\mathcal{N}(\psi)$, so it follows from (3) that

$$
(d \psi \otimes d \psi)^{o} \equiv 0
$$


on $\mathcal{N}(\psi)$. This is equivalent to

$$
d \psi \otimes d \psi=\frac{1}{n}|d \psi|_{g}^{2} \cdot g
$$

Now, the right-hand side has rank $n$ whenever $|d \psi|_{g} \neq 0$. On the other hand, the left-hand side has rank $\leq 1$. The only way the equality is possible if both side are identically zero on $\mathcal{N}(\psi)$, i.e. if

$$
\left.d \psi\right|_{\mathcal{N}(\psi)} \equiv 0 .
$$

However, it is well-known (see e.g. [Che76, Han94, HHL98, HHHN99]) that the intersection of the nodal and critical sets of $\psi$ has locally finite Hausdorff $(n-2)$ dimensional measure, and so (4) is impossible for non-zero $\psi$. This contradiction finishes the proof of Proposition 4.1.

An immediate consequence of the proof of Proposition 4.1 and Proposition 3.1 is the following corollary.

Corollary 4.2. Let $g_{0}$ be a Yamabe metric on $M, g_{0} \in \mathcal{M}_{0, m}(M) \cap \mathcal{M}_{0, m+1}(M)^{c}$. Let $h=c_{n} \psi\left(2 \psi \stackrel{\circ}{\nabla}^{2} \psi-\psi^{2}\right.$ Ric $)+\left(2 c_{n}-1\right)(d \psi \otimes d \psi)^{\circ}$, where $\psi=\psi_{g_{0}}$ is a nonzero eigenfunction of $Y_{g_{0}}$ with eigenvalue 0 . Consider the perturbation $g(t)=g_{0}+t$. Then for every $\epsilon>0$, there exists $|t| \leq \epsilon$ such that $g(t) \in \mathcal{M}_{0, m}^{c}$.

To complete the proof of Theorem 2.3, we need to prove the following

Claim 4.3. Proposition 4.1 implies Theorem 2.3.

Proof of Claim 4.3. Since $\mathcal{T}_{0,1}(M)$ is a closed subspace of $\mathcal{T}(M)$, its complement $\mathcal{T}_{0,1}(M)^{c}$ is clearly open in $\mathcal{T}(M)$. We thus need to show that $\mathcal{T}_{0,1}(M)^{c}$ is dense in $\mathcal{T}(M)$. We shall show that $\mathcal{M}_{0,1}(M)^{c}$ is dense in $\mathcal{M}(M)$. Let $g_{0}$ be a metric on $M$. It suffices to show that $\mathcal{M}_{0,1}(M)^{c}$ is dense in some neighbourhood $U$ of $g_{0}$ in $\mathcal{M}(M)$. If $g_{0} \in \mathcal{M}_{0,1}(M)^{c}$, we are done, so we can assume that $g_{0} \in \mathcal{M}_{0,1}(M)$.

The proof proceeds by induction on the dimension $m$ of $E_{0}$. We note that $m$ is finite for any $g_{0}$, and that Corollary 4.2 was proved for arbitrary $m$. First, let $m=1$, meaning 0 is a simple eigenvalue of $Y_{g_{0}}$. By Corollary 4.2, we know that one can choose a curve of metrics $g(t)$, real analytic in $t$, such that $g(0)=g_{0}$ and $g(t) \notin \mathcal{M}_{0,1}$ for arbitrary small $t .^{2}$ Hence, the proof in case $m=1$ is complete.

Next, assume that we have shown that $\mathcal{M}_{0,1}(M)^{c}$ is dense in any neighborhood $U$ of any metric $g_{0} \in \mathcal{M}_{0,1}(M)$ such that zero is an eigenvalue of $Y_{g_{0}}$ with the multiplicity at most $m-1$; we would like to prove the corresponding statement for a metric $g_{0}$ such that 0 is an eigenvalue of $Y_{g_{0}}$ with multiplicity exactly $\mathrm{m}$. By Corollary 4.2, there exists a small perturbation that decreases the multiplicity $m$ of 0 as an eigenvalue of $Y_{g}$. By the inductive hypothesis, it follows that for any neighborhood $U$ of $g_{0}$, there exists a metric $g_{1} \in U$, such that 0 is an eigenvalue of $Y_{g_{1}}$ with multiplicity $\leq m-1$, and in a suitable neighborhood $V$ of $g_{1}$ (which can be chosen to satisfy $V \subset U)$, have a nonempty intersection with $\mathcal{M}_{0,1}(M)^{c}$. This

\footnotetext{
${ }^{2}$ It will then follow that (in the notation of [Tey99]), the space of conformal structures corresponding to metrics in $\mathcal{M}_{0,1}$ is of meager codimension 1 in the space of all conformal structures; we leave the details of the argument to the reader.
} 
completes the proof of the Claim 4.3, and hence also of Theorems 2.3 and 1.1.

\section{Negative eigenvalues of the CONFormal Laplacian}

In [CGJP14, CGJP13, ES14] the authors showed that the number of negative eigenvalues of the conformal Laplacian cannot be uniformly bounded above on any compact manifold $M$ of dimension $n \geq 3$. Accordingly, it seems interesting to consider sequences of metrics $g_{k}$ on $M$ where the number of negative eigenvalues of $Y_{g_{k}}=-\Delta_{g_{k}}+c_{n} R_{g_{k}}$ is growing.

It is known that the set of metrics $g_{k}$ on a manifold $M$ of dimension $n \geq 3$ is pre-compact in Gromov-Hausdorff topology if it satifies either condition 5.1 or condition 5.2 below:

Condition 5.1. The volume $\operatorname{Vol}\left(M, g_{k}\right) \leq V<\infty$ is bounded above; the injectivity radius $\operatorname{inj}\left(M, g_{k}\right) \geq r>0$ is bounded from below; the Ricci curvature $\operatorname{Ric}\left(M, g_{k}\right) \geq$ $-a^{2}$ is bounded from below.

Condition 5.2. The diameter $\operatorname{diam}\left(M, g_{k}\right) \leq D<\infty$ is bounded above; the Ricci curvature $\operatorname{Ric}\left(M, g_{k}\right) \geq-a^{2}$ is bounded from below.

Consider a sequence of metrics $\tilde{g}_{k}$ on a fixed Riemannian manifold such that the number of negative eigenvalues of the conformal Laplacian $Y_{\tilde{g}_{k}}$ goes to infinity. It is natural to choose a unique Yamabe representative $g_{k}$ in the conformal class $\left[\tilde{g}_{k}\right]$; the scalar curvature of $g_{k}$ is constant and equal to -1 ; the number of negative eigenvalues of $Y_{g_{k}}$ and $Y_{\tilde{g}_{k}}$ are equal.

Proposition 5.3. The sequence $g_{k}$ cannot satisfy the pre-compactness condition 5.1; nor can it satisfy the condition 5.2.

Proof of Proposition 5.3: The result follows from [Bus82, Thm. 6.2] and [Gro99, Appendix C]. Indeded, since $g_{k}$ is Yamabe, the number of negative eigenvalues of $Y_{g_{k}}$ is equal to the number $N\left(\frac{n-2}{4(n-1)} ; g_{k}\right)$ of eigenvalues of the Laplacian $-\Delta_{g_{k}}$ that are less than $(n-2) /(4(n-1))$. Assuming $g_{k}$ satisfies 5.1, it follows from [Bus82, Thm. 6.2] that $N\left(\frac{n-2}{4(n-1)} ; g_{k}\right) \leq C_{1}<\infty$ where the constant $C_{1}$ only depends on $V, r, n, \delta$. Similarly, assuming $g_{k}$ satisfies Condition 5.2, it follows from Gromov's result in [Gro99, Appendix C] that $N\left(\frac{n-2}{4(n-1)} ; g_{k}\right) \leq C_{2}<\infty$ where the constant $C_{2}$ only depends on $D, n, a$. These contradict the assumption on the number of negative eigenvalues of $Y_{g_{k}}$.

Proposition 5.3 shows that sequences of metrics with increasing number of negative eigenvalues of $Y_{g_{k}}$ cannot stay in the "thick" part of $\mathcal{M}$ satisfying natural pre-compactness conditions 5.1 or 5.2 , and thus we cannot use those conditions to choose a convergent subsequence of metrics. On the other hand, we remark that on certain high-dimensional manifolds (cf. [GL80]) there exist infinitely many connected components of the set of metrics with positive scalar curvature. Accordingly, the sequence of metrics can diverge but the number of negative eigenvalues of $Y$ can stay equal to 0 .

5.1. Example: product of a surface with another manifold. We consider (a slight modification of) one of the examples discussed in [CGJP14, §4]. Let $M$ be a manifold of dimension $d \geq 2$, and let $\Sigma$ be a Riemann surface of genus 
$\gamma \geq 2$. Assume that $M$ admits a metric with positive scalar curvature, and fix a Yamabe metric $G$ on $M$ with scalar curvature $R_{G}>0$. Fix $\epsilon>0$. By a result of Buser [Bus77, Theorem 4], for every $k \geq 1$, there exists a hyperbolic metric $h_{k}$ on $\Sigma$ such that the hyperbolic Laplacian $-\Delta_{h_{k}}$ has at least $k$ eigenvalues in the interval $(1 / 4,1 / 4+\epsilon)$. Choose $k$ of those eigenvalues and denote them by $1 / 4<\lambda_{k, 1} \leq \lambda_{k, 2} \leq \ldots \leq \lambda_{k, k}<1 / 4+\epsilon$. Denote the corresponding eigenfunctions by $u_{k, j}, 1 \leq j \leq k$.

Consider the product metric $g_{k}:=\left(G \otimes t^{-1} h_{k}\right)$ on $M \times \Sigma$, where $t$ is a positive constant to be chosen later. It is easy to show that the scalar curvature of $g_{k}$ is equal to $R_{G}-2 t$ for all $k$ (the Gauss curvature of $\left(\Sigma, h_{k}\right)$ is equal to -1 ). If we choose

$$
t>R_{G} / 2
$$

then the scalar curvature of $g_{k}$ will be negative.

Denote the coordinates on $M \times \Sigma$ by $(x, y)$. Then the conformal Laplacian is given by

It follows that

$$
Y_{g_{k}}=-\Delta_{G, x}-t \Delta_{h_{k}, y}+\frac{d}{4(d+1)}\left(R_{G}-2 t\right)
$$

$$
Y_{g_{k}} u_{j, k}=\left(t \lambda_{j, k}+\frac{d\left(R_{G}-2 t\right)}{4(d+1)}\right) u_{j, k} .
$$

We would like to choose $t$ so that the eigenvalues $t \lambda_{j, k}+\frac{d\left(R_{G}-2 t\right)}{4(d+1)}$ are all negative. Since $\lambda_{j, k}<1 / 4+\epsilon$ by assumption on $h_{k}$, it suffices to choose $t$ so that

$$
\frac{d\left(2 t-R_{G}\right)}{4 t(d+1)}>\frac{1}{4}+\epsilon
$$

This can be rewritten as

$$
\left(\frac{d}{d+1}\right)\left(1-R_{G} / 2 t\right)>1 / 2+2 \epsilon
$$

A straightforward calculation shows that this is equivalent to choosing

$$
t<R_{G} \cdot \frac{d}{d-1-4 \epsilon(d+1)}
$$

The inequalities (5) and (6) are compatible provided $d /(d-1-4 \epsilon(d+1))>1 / 2$, which is easy to achieve by choosing $\epsilon$ small enough. It follows that the functions $u_{j, k}(y)$ will be eigenfunctions of $Y_{g_{k}}$ with negative eigenvalues.

After rescaling $g_{k}$ by $\left(2 t-R_{G}\right)$, we can make the scalar curvature $R_{g_{k}} \equiv-1$. Note that the rescaling does not depend on $k$. It is well-known that as the number of eigenvalues of $-\Delta_{h_{k}}$ in $(1 / 4,1 / 4+\epsilon)$ increases, the injectivity radius of the metric $h_{k}$ goes to 0 , and $h_{k}$ leaves the "thick" part of the moduli space $\mathcal{M}_{\gamma}$ of the hyperbolic metrics on $\Sigma .^{3}$ Accordingly, the injectivity radius of $\left(M \times \Sigma, g_{k} /\left(2 t-R_{G}\right)\right)$ also goes to 0 . The moduli space $\mathcal{M}_{\gamma}$ can be compactified by adding surfaces with cusps; the sequence $h_{k}$ will then have a convergent subsequence in $\overline{\mathcal{M}_{\gamma}}$, and the sequence $\left(M \times \Sigma, g_{k} /\left(2 t-R_{G}\right)\right)$ will also have a convergent subsequence.

It seems interesting to better understand under what circumstances a sequence of metrics $g_{k}$, with increasing number of negative eigenvalues of $Y_{g_{k}}$, can be made to

\footnotetext{
${ }^{3}$ See e.g. [Bus92]; for finer asymptotics of small eigenvalues we refer to [Bat98] and references therein.
} 
converge in a suitable completion of the moduli space $\mathcal{R}(M)$ of conformal structures on $M$.

\section{ACKNOWLEDGEMENTS}

The authors wish to thank Yaiza Canzani, Raphaël Ponge, Peter Sarnak and Richard Schoen for stimulating discussions about this problem. The authors would like to thank the organizers of the workshop "Shape optimization and spectral geometry" at International Centre for Mathematical Sciences in Edinburgh, where part of this research was conducted. A.H. gratefully acknowledges the support and hospitality of the Centre de recherche mathématiques in Montreal and the Max-Planck Institute for Mathematics in Bonn. D. J. gratefully acknowledges the hospitality of the Department of Mathematics at the University College London, as well as the Department of Mathematics at the University of Auckland. The authors would like to thank the anonymous referee for useful remarks about the paper.

\section{REFERENCES}

[Bat98] Ph. Batchelor. Dérivée des petites valeurs propres des surfaces de Riemann. Comment. Math. Helv., 73(3):337-352, 1998.

[BD03] C. Bär and M. Dahl. Small eigenvalues of the conformal Laplacian. Geom. Funct. Anal., 13(3):483-508, 2003.

[BE69] M. Berger and D. Ebin. Some decompositions of the space of symmetric tensors on a Riemannian manifold. J. Differential Geometry, 3:379-392, 1969.

[Ber70] M. Berger. Quelques formules de variation pour une structure riemannienne. Ann. Sci. École Norm. Sup. (4), 3:285-294, 1970.

[Ber73] M. Berger. Sur les premières valeurs propres des variétés riemanniennes. Compositio Math., 26:129-149, 1973.

[Bus77] P. Buser. Riemannsche Flächen mit Eigenwerten in (0, 1/4). Comment. Math. Helv., 52(1):25-34, 1977.

[Bus82] P. Buser. A note on the isoperimetric constant. Ann. Sci. École Norm. Sup. (4), 15(2):213-230, 1982.

[Bus92] P. Buser. Geometry and spectra of compact Riemann surfaces, volume 106 of Progress in Mathematics. Birkhäuser Boston, Inc., Boston, MA, 1992.

[BW80] D. Bleecker and L. Wilson. Splitting the spectrum of a Riemannian manifold. SIAM J. Math. Anal., 11(5):813-818, 1980.

[Can14] Y. Canzani. On the multiplicity of eigenvalues of conformally covariant operators. Ann. Inst. Fourier (Grenoble), 64(3):947-970, 2014.

[CGJP13] Y. Canzani, R. Gover, D. Jakobson, and R. Ponge. Nullspaces of conformally invariant operators. Applications to $Q_{k}$-curvature. Electron. Res. Announc. Math. Sci., 20:4350, 2013.

[CGJP14] Y. Canzani, R. Gover, D. Jakobson, and R. Ponge. Conformal invariants from nodal sets. I. Negative eigenvalues and curvature prescription. Int. Math. Res. Not. IMRN, (9):2356-2400, 2014. With an appendix by Gover and Andrea Malchiodi.

[Che76] S.Y. Cheng. Eigenfunctions and nodal sets. Comment. Math. Helv., 51(1):43-55, 1976.

[Dah03] M. Dahl. Dirac eigenvalues for generic metrics on three-manifolds. Ann. Global Anal. Geom., 24(1):95-100, 2003.

[Dah08] M. Dahl. On the space of metrics with invertible Dirac operator. Comment. Math. Helv., 83(2):451-469, 2008.

[DWW05] X. Dai, X. Wang, and G. Wei. On the stability of Riemannian manifold with parallel spinors. Invent. Math., 161(1):151-176, 2005.

[ES14] S. El Sayed. Second eigenvalue of the Yamabe operator and applications. Calc. Var. Partial Differential Equations, 50(3-4):665-692, 2014.

[FM77] A. Fischer and J. Marsden. The manifold of conformally equivalent metrics. Canad. J. Math., 29(1):193-209, 1977. 
[FM96] A. Fischer and V. Moncrief. The structure of quantum conformal superspace. In Global structure and evolution in general relativity (Karlovassi, 1994), volume 460 of Lecture Notes in Phys., pages 111-173. Springer, Berlin, 1996.

[FM97] A. Fischer and V. Moncrief. Hamiltonian reduction of Einstein's equations of general relativity. Nuclear Phys. B Proc. Suppl., 57:142-161, 1997. Constrained dynamics and quantum gravity 1996 (Santa Margherita Ligure).

[GL80] M. Gromov and H. B. Lawson. The classification of simply connected manifolds of positive scalar curvature. Ann. of Math. (2), 111(3):423-434, 1980.

[Gro99] M. Gromov. Metric structures for Riemannian and non-Riemannian spaces, volume 152 of Progress in Mathematics. Birkhäuser Boston, Inc., Boston, MA, 1999. Based on the 1981 French original [ MR0682063 (85e:53051)], With appendices by M. Katz, P. Pansu and S. Semmes, Translated from the French by Sean Michael Bates.

[Han94] Q. Han. Singular sets of solutions to elliptic equations. Indiana Univ. Math. J., 43(3):983-1002, 1994

[HHHN99] R. Hardt, M. Hoffmann-Ostenhof, T. Hoffmann-Ostenhof, and N. Nadirashvili. Critical sets of solutions to elliptic equations. J. Differential Geom., 51(2):359-373, 1999.

[HHL98] Q. Han, R Hardt, and F Lin. Geometric measure of singular sets of elliptic equations. Comm. Pure Appl. Math., 51(11-12):1425-1443, 1998.

[KS60] K. Kodaira and D. C. Spencer. On deformations of complex analytic structures. III. Stability theorems for complex structures. Ann. of Math. (2), 71:43-76, 1960.

[KW75] J. Kazdan and F. Warner. Scalar curvature and conformal deformation of Riemannian structure. J. Differential Geometry, 10:113-134, 1975.

[Loh96] J. Lohkamp. Discontinuity of geometric expansions. Comment. Math. Helv., 71(2):213228, 1996.

[Mai97] S. Maier. Generic metrics and connections on Spin- and Spin ${ }^{c}$-manifolds. Comm. Math. Phys., 188(2):407-437, 1997.

[Pon] R. Ponge. Generic multiplicity of eigenvalues and resonances. In preparation.

[Rel69] F. Rellich. Perturbation theory of eigenvalue problems. Assisted by J. Berkowitz. With a preface by Jacob T. Schwartz. Gordon and Breach Science Publishers, New YorkLondon-Paris, 1969.

[Tey99] M. Teytel. How rare are multiple eigenvalues? Comm. Pure Appl. Math., 52(8):917934, 1999.

Department of Mathematics, The University of Auckland, Private Bag 92019, AuckLAND 1142, NEW ZEALAND.

E-mail address: r.gover@auckland.ac.nz

Max-Planck Institute for Mathematics Vivatsgasse 7, 53111 Bonn, Germany

E-mail address: hassannezhad@mpim-bonn.mpg.de

Department of Mathematics and Statistics, McGill University, 805 Sherbrooke Str. West, Montréal QC H3A 2K6, Canada.

E-mail address: jakobson@math.mcgill.ca

Department of Mathematics and Statistics, University of Reading, Whiteknights, PO Box 220, Reading RG6 6AX, United Kingdom

E-mail address: M.Levitin@reading.ac.uk 